\title{
Nonidentical relations of skew-symmetric forms: Generation of closed exterior forms. Discrete transitions. Connection between field-theory equations and nonidentical relations
}

\author{
L.I. Petrova ${ }^{1 *}$ \\ ${ }^{1}$ Moscow State University, Department of Computational Mathematics and Cybernetics, Russia \\ *Corresponding authorE-mail: ptr@cs.msu.su
}

\begin{abstract}
Nonidentical relations of skew-symmetric differential forms, which basis are non-integrable deforming manifolds follow from differential equations. From nonidentical relations closed exterior forms are obtained. The process of obtaining closed exterior forms describes the discrete transitions and the emergence of structures and observable formations such as waves, vertices, and turbulent pulsations.

It is shown that the field theory equations (by Schroedinger, Maxwell, Einstein and others) turns to be nonidentical relations, obtained from the mathematical physics equations for material media such as the cosmologic systems, the systems of charged particles and others.
\end{abstract}

Keywords: Degenerate transformation; discrete transitions; nonidentical and identical relations; non-integrable manifolds; skew-symmetric forms.

\section{Introduction}

Nonidentical relations of skew-symmetric differential forms disclose unique capabilities of skew-symmetric ones and their role in mathematics, mathematical physics and field theory. The properties of closed exterior and dual forms, namely, invariance, covariance, conjugace, and duality, either explicitly or implicitly appear in all invariant mathematical formalisms. This enables one to see an internal connection between various branches of mathematics.

However, the theory of closed exterior forms cannot be completed without an answer to a question of how the closed exterior forms emerge.

It turns out that the closed inexact exterior forms are generated by the skew-symmetric forms obtained from the differential equations describing by any processes. These skew-symmetric forms possess evolutionary properties since, in contrast to exterior forms, the nonintegrable deforming manifold is their basis.

In the paper it is shown that the process of generating closed exterior forms, the tool of which are nonidentical relations, describe the evolutionary processes and the processes of various structures emergence, which is impossible to describe within the framework of another mathematical formalisms.

In the paper, it is disclosed an unique physical sense of the nonidentical relations obtained from the equations of mathematical physics that describe material media such as thermodynamic, gas-dynamic, cosmic systems, the systems of charged particles and so on. From these equations one can obtain the nonidentical evolutionary relation for functionals such as entropy, action functional, Einstein's function, and so on, which, as it is shown, possess a duality. They are functionals of the material media state and (as it is well known) the field-theory functionals. On one hand, such a nonidentical relation describes the material media state, evolutionary processes in material media and the processes of emergence of physical structures and such formations like waves, vortices and turbulent pulsations. And on other hand, this relation discloses the connection between the field-theory equations and the equations of mathematical physics. It is shown that the field theory equations (by Schroedinger, Maxwell, Einstein and others) which solutions are differentials, namely, closed inexact exterior differential forms, turns to be nonidentical relations (relations, from which closed exterior forms are obtained) of the equations of mathematical physics for material media.

In Section 1 we present some information (being necessary for further outlining) concerning the properties of skew-symmetric exterior and evolutionary forms. Specific features of nonidentical relations and degenerate transformation of the evolutionary differential forms are described. The process obtaining identical relations and the process of generating closed inexact exterior differential forms are described.

In Section 2 the unique properties of the nonidentical evolutionary relation obtained from the equations of mathematical physics, which describe material media are presented.

Such a relation reveals the hidden properties of the mathematical physics equations that enables one to describe not only the variation of physical quantities, but also to describe the evolutionary processes in material media such as the emergence of various structures and observable formations.

In Section 3 the connection between the field-theory equations and the nonidentical relation of the mathematical physics equations is 
shown.

\section{Some properties of skew-symmetric forms}

(From papers [1-4] one can get more detail information concerning the skew-symmetric differential forms).

\subsection{Closed exterior differential forms}

The exterior differential form of the degree $p$ ( $p$-form) can be written as [3-5]:

$$
\theta^{p}=\sum_{i_{1} \ldots i_{p}} a_{i_{1} \ldots i_{p}} d x^{i_{1}} \wedge d x^{i_{2}} \wedge \cdots \wedge d x^{i_{p}} \quad 0 \leq p \leq n
$$

Here $a_{i_{1} \ldots i_{p}}$ is the function of independent variables $x^{1}, \ldots, x^{n}, n$ is the space dimension, and $d x^{i}, d x^{i} \wedge d x^{j}, d x^{i} \wedge d x^{j} \wedge d x^{k}, \ldots$ is the local basis subject to the condition of skew-symmetry:

$$
\begin{aligned}
& d x^{i} \wedge d x^{i}=0 \\
& d x^{i} \wedge d x^{j}=-d x^{j} \wedge d x^{i} \quad i \neq j
\end{aligned}
$$

The exterior form differential $\theta^{p}$ is expressed by the formula

$$
d \theta^{p}=\sum_{i_{1} \ldots i_{p}} d a_{i_{1} \ldots i_{p}} \wedge d x^{i_{1}} \wedge d x^{i_{2}} \cdots \wedge d x^{i_{p}}
$$

The form called as a closed one if its differential equals to zero:

$$
d \theta^{p}=0
$$

From condition (4) one can see that the closed form is a conservative quantity. This means that such a form can correspond to the conservation law (for physical fields).

If the form be closed only on pseudostructure, i.e. this form is a closed inexact one, the closure condition can be written as

$$
d_{\pi} \theta^{p}=0
$$

In this case the pseudostructure $\pi$ obeys the condition

$$
d_{\pi}^{*} \theta^{p}=0
$$

here ${ }^{*} \theta^{p}$ is the dual form.

From conditions (5) and (6) one can see that the dual form (pseudostructure) and closed inexact form (conservative quantity) describe a conservative object that can also correspond to some conservation law. (It appears that the closed inexact exterior and dual forms describe a structure with conservative quantity. Such structures made up physical fields and pseudometric and metric manifolds.)

\section{Properties of closed exterior forms}

Invariance of closed exterior forms. Non-degenerate transformations

The fundamental properties of exterior differential forms are connected with the fact that any closed form is a differential. An exact form is, by definition, a differential. In this case the differential is total. A closed inexact form is a differential too. The closed inexact form is an interior (on pseudostructure) differential, that is

$$
\theta_{\pi}^{p}=d_{\pi} \theta^{p-1}
$$

Since a closed form is a differential, it is obvious that a closed form will turn out to be invariant under all non-degenerate transformations that conserve a differential.

The non-degenerate transformations that conserve the differential are one of fundamental methods in the theory of exterior differential forms (below it will be shown that degenerate transformations appear in the mathematical apparatus of the evolutionary forms). Unitary transformations ( 0 -forms), the tangent and canonical transformations (1-forms), gradient and gauge transformations (2-forms), and so on are examples of such transformations. These are the gauge transformations for spinor, scalar, vector and tensor (3-form) fields. Covariance of a dual form is directly connected with the invariance of an exterior closed form.

Invariant properties of closed exterior forms and covariance of a dual form explicitly or implicitly manifest themselves essentially in all invariant mathematical formalisms and formalisms of field theory, such as the Hamilton formalism, tensor calculus, group theory, YangMills theory and others.

\section{Identical relations of exterior differential forms}

Closure of exterior differential forms and dual forms, and hence their invariance and covariance, results from the conjugace of the elements of exterior or dual forms, elements of the form differential, exterior and dual forms and others.

Since the conjugace represents a certain connection between two operators or mathematical objects, it is evident that relations can be used to express conjugace mathematically. A relation may be either identical or nonidentical.

The basis of the mathematical apparatus of exterior differential forms comprises identical relations. (Below the nonidentical relations will be discussed, and it will be shown that identical relations for exterior differential forms are obtained from nonidentical relations by the degenerate transformations.)

The identical relations express the fact that the differential of exterior form is a closed exterior form of any degree $p$.

In general form such an identical relation can be written as

$$
d_{\pi} \phi=\theta_{\pi}^{p}
$$

In this relation the form in the right-hand side has to be a closed one. (As it will be shown below, the identical relations are satisfied on pseudostructures).

[The importance of the identical relations for exterior differential forms is manifested by the fact that practically in all invariant branches of physics, mechanics and thermodynamics one faces such identical relations. One can present the following examples: (a) the Poincare invariant $d s=-H d t+p_{j} d q_{j}$, (b) the second principle of thermodynamics $d S=(d E+p d V) / T$, (c) the conditions on characteristics in the theory of differential equations, and so on. The relations expressed in terms of integral, tensor and derivatives analogues to differential forms are also identical relations of exterior skew-symmetric forms forms [3]

Differential-geometrical structures and their physical meaning The closed dual form and associated closed inexact exterior form made up a differential-geometrical structure that describes a pseudostructure with conservative quantity. (A closed dual form describes a pseudostructure. And a closed exterior form, as it is known, describes a conservative quantity, since the differential of closed form is equal to zero).

Such a differential-geometrical structure describes a conservative object (a pseudostructure with conservative quantity). As it will be shown below such differential-geometrical structures describe physical structures on which conservation laws are fulfilled.

The properties of closed exterior forms (invariance, conjugace, duality, and so on) manifest themselves in various branches of mathematics such as algebra, differential geometry, the theory of functions of complex variables, tensor analysis, differential and integral calculus. This discloses an internal connection between various mathematical formalisms.

However, the theory of closed exterior forms cannot be completed without an answer to a question of how the closed exterior forms emerge.

It turns out that closed exterior forms are obtained from differential equations describing any processes. 
However, here there is a certain peculiarity. Closed exterior skewsymmetric forms cannot be directly obtained from differential equations. They are realized only in evolutionary process.

When studying the integrability of differential equations it follows an nonidentical relation that contains the skew-symmetric differential forms which possess the evolutionary properties since they are defined on deforming manifold. As the result of the process made up by nonidentical relation the closed inexact exterior forms are obtained from such evolutionary skew-symmetric differential forms. As it will be shown, the process of extracting closed exterior forms from evolutionary forms describes the generation of various invariant structures, discrete transitions, emergence of physical structures, and so on.

\subsection{Evolutionary skew-symmetric differential forms}

The evolutionary skew-symmetric differential forms are obtained from differential equations that describe any processes.

A radical distinction between the evolutionary forms and the exterior ones consists in the fact that evolutionary differential forms, in distinction from the exterior differential form, are defined on deforming non-integrable manifolds. Examples of non-integrable manifolds, on which the evolutionary skew-symmetric differential forms are defined, are the tangent manifolds of differential equations, the Lagrangian manifolds, the manifolds made up by trajectories of material medium particles, and so on. These are manifolds with unclosed metric forms. The metric form differential, and correspondingly its commutator, are nonzero. (The commutators of metric forms of such manifolds describe the manifold deformation, namely, torsion, curvature and others). (In Appendix 1 some properties of such manifolds are presented and it is shown the distinction between exterior and evolutionary forms that depends on the properties of metric forms of the manifold on which these skew-symmetric forms are defined.)

The evolutionary form can be written in a way similar that for exterior differential form. However, in distinction from the exterior form differential, an additional term will appear in the evolutionary form differential. This is due to the fact that the evolutionary form basis changes since such a form is defined on deforming manifold.

The evolutionary form differential takes the form

$d \boldsymbol{\theta}^{p}=\sum_{i_{1} \ldots i_{p}} d a_{i_{1} \ldots i_{p}} \wedge d x^{i_{1}} \wedge d x^{i_{2}} \cdots \wedge d x^{i_{p}}+\sum_{i_{1} \ldots i_{p}} a_{i_{1} \ldots i_{p}} d\left(d x^{i_{1}} \wedge d x^{i_{2}} \ldots\right.$

where the second term is connected with the basis differential of nonzero value: $d\left(d x^{i_{1}} \wedge d x^{i_{2}} \wedge \cdots \wedge d x^{i_{p}}\right) \neq 0$. (For the exterior form defined on integrable manifold one has $d\left(d x^{i_{1}} \wedge d x^{i_{2}} \wedge \cdots \wedge d x^{i_{p}}\right)=$ $0)$.

\section{The specific feature and properties of evolutionary forms}

\section{Nonclosure of the evolutionary differential forms}

The specific feature of evolutionary forms, i.e. skew-symmetric forms defined on deforming manifolds, consists in the fact that evolutionary forms are unclosed ones. Since the basis of evolutionary form changes, the evolutionary form differential includes the nonvanishing differential of manifold metric form emerged when differentiating the basis. Therefore, the evolutionary form differential cannot be equal to zero. Hence, the evolutionary form, in contrast to the case of exterior form, cannot be closed. (In Appendix 1 the peculiarity of skew-symmetric forms defined on nonintegrable manifold are demonstrated by the example of a skew-symmetric form of first-degree.)

Since the evolutionary differential forms are unclosed, the theory of evolutionary differential forms includes some unconventional elements, such as nonidentical relations, self-variation and degenerate transformations that allow to describe the generation of closed inexact exterior forms.

\section{Nonidentical relations of evolutionary differential forms}

Above it was shown that the identical relations lie at the basis of the mathematical apparatus of exterior differential forms.

In contrast to this, nonidentical relations lie at the basis of the mathematical apparatus of evolutionary differential forms.

The identical relations establish exact correspondence between the quantities (or objects) involved into this relation. This is possible in the case when the quantities involved into the relation are measurable ones.

In the nonidentical relations one of the quantities is unmeasurable. The nonidentical relations of evolutionary forms can be written as

$$
d \phi=\omega^{p}
$$

Here $\omega^{p}$ is the $p$-degree evolutionary form.

In the left-hand side of this relation it stands the form differential, i.e. the closed form, which is an invariant and measurable quantity. In the right-hand side it stands the unclosed evolutionary form, which is not a measurable quantity. Such a relation cannot be identical.

Nonidentical relations are evolutionary relations because they include the evolutionary form.

One can see the difference of relations for exterior forms and evolutionary ones. In the right-hand side of identical relation (see relation (7)) it stands a closed exterior form, whereas the form in the righthand side of nonidentical relation (9) is an unclosed one.

Nonidentical relations are obtained when describing any processes. [A relation of such type is obtained, for example, when analyzing the integrability of the partial differential equation. The equation is integrable if it can be reduced to the form $d \phi=d U$. However, it turns out that, if the equation is not subject to an additional condition (the integrability condition), it is reduced to the form (9), where $\omega^{p}$ is an unclosed form and it cannot be written as a differential. Here it should be emphasized that the nonidentity of relation (9) does not mean that the mathematical description of the process is not sufficiently accurate. The nonidentity of the relation means that the function derivatives, which values correspond to the real values in physical processes, cannot be consistent. For physical processes the expressions for these derivatives are usually obtained independently of one another. And they are not conjugated, that is they do not made up a differential.]

\section{S,elfvariation of the evolutionary nonidentical relation}

The evolutionary nonidentical relation is self-varying, because, firstly, it is nonidentical, namely, it contains two objects one of which appears to be unmeasurable, and, secondly, it is an evolutionary relation, namely, a variation of any object of the relation in some process leads to variation of another object and, in turn, a variation of the latter leads to variation of the former. Since one of the objects is an unmeasurable quantity, the other cannot be compared with the first one, and hence, the process of mutual variation cannot end. This process is governed by the evolutionary form commutator. The significance of the evolutionary relation selfvariation consists in the fact that in such a process it can be realized the conditions under which the closed inexact forms can be obtained from the evolutionary form. These are conditions of degenerate transformation.

\section{Degenerate transformations.}

Unique significance of the evolutionary skew-symmetric forms consists in the fact that they can generate closed exterior forms, which lie at the basis of many invariant mathematical formalisms.

This proceeds with the help of degenerate transformation.

From the evolutionary unclosed skew-symmetric form, which differential is nonzero, one can obtain a closed exterior form with a differential being equal to zero only under degenerate transformation, namely, under a transformation that does not conserve the 
differential. (The Legendre transformation is an example of such a transformation.)

Degenerate transformations can take place under additional conditions, which are caused by degrees of freedom. The vanishing of such functional expressions as determinants, Jacobians, Poisson's brackets, residues, and others corresponds to these additional conditions. Vanishing of these functional expressions is a closure condition for a dual form. (It can be noted that, when solving many problems, the condition that the above listed functional expressions must be nonzero is imposed. This leads to that the solutions, which are not invariant but have physical meaning, are not accounted for, that is, the solutions, which describe evolutionary processes, are ignored.)

As it was already noted, the additional conditions can be realized under a selfvariation of nonidentical evolutionary relation.

\subsection{Generation of closed inexact exterior differential forms}

A distinction of the evolutionary form from the closed exterior form consists in the fact that the evolutionary differential form is defined on deforming non-integrable manifold, i.e., on the manifold with unclosed metric forms, but the closed exterior form can be defined only on integrable manifold, namely, on the manifold with closed metric forms.

Hence, it follows that a closed exterior form can be obtained from the evolutionary form only under degenerate transformation, when the transition from the manifold with unclosed metric forms (whose differential is nonzero) to integrable structures with closed metric forms (for which the differential is zero) takes place.

For this reason the transition from an evolutionary form to a closed exterior form proceeds only when the differential or commutator of the metric form manifold, on which the evolutionary form is defined, becomes equal to zero.

The realization of closure of the manifold metric form is a realization of the dual form closure. And this leads to realization of pseudostructure and the skew-symmetric form closure on pseudostructure, i.e., the realization of closed inexact exterior form.

As it was already noted, the conditions of degenerate transformation are realized when the functional expressions such as Jacobians, determinants, the Poisson brackets, residues, and others vanish. The conditions of degenerate transformation specify the integral structures (pseudostructures), namely, the characteristics of differential equations (the determinant of coefficients at the normal derivatives vanishes), the singular points (Jacobian is equal to zero) and so on. [The Cauchy-Riemann conditions, the characteristic relations, the canonical relations, the Bianchi identities, and others are examples of the conditions of degenerate transformations (vanishing the dual form differential).]

Conditions of degenerate transformation can be realized under selfvariation of a nonidentical relation, for example, if there exist any symmetries of the evolutionary or dual form coefficients or their derivatives. (When describing material media this can be caused by an availability of any degrees of freedom of material system.) This can take place only discretely rather than identically.

Under degenerate transformation the following transition proceeds: $d \omega^{p} \neq 0 \rightarrow$ (a degenerate transformation) $\rightarrow d_{\pi} \omega^{p}=0, d_{\pi}^{*} \omega^{p}=0$ The realization of the conditions $d_{\pi}^{*} \omega^{p}=0$ and $d_{\pi} \omega^{p}=0$ means that it is realized the closed dual form ${ }^{*} \omega^{p}$, which describes some structure $\pi$ (which is a pseudostructure with respect to its metric properties), and the closed exterior (inexact) form $\omega_{\pi}^{p}$, which basis is a pseudostructure, is obtained.

Thus, if the conditions for degenerate transformation are realized, from an unclosed evolutionary form one can obtain the differential form closed on pseudostructure, which is a closed inexact exterior form.

On the pseudostructure $\pi$ nonidentical evolutionary relation (9) trans- forms into the relation

$$
d_{\pi} \phi=\omega_{\pi}^{p}
$$

which appears to be an identical relation. Indeed, since the form $\omega_{\pi}^{p}$ is a closed one, on the pseudostructure this form turns out to be a differential of some differential form. In other words, this form can be written as $\omega_{\pi}^{p}=d_{\pi} \theta$. Relation (9) is now written as $d_{\pi} \phi=d_{\pi} \theta$ There are differentials in the left-hand and right-hand sides of this relation. This means that the relation (10) is an identical one.

It can be shown that all identical relations of the exterior differential form theory are obtained from nonidentical relations (that contain the evolutionary forms) by applying degenerate transformations. In this case the evolutionary relation itself remains to be nonidentical one, since the evolutionary form remains to be unclosed.

Under degenerate transformation the transition from non-integrable manifold to integral stricture occurs. Mathematically, the degenerate transformation is realized as a transition from one frame of reference to another (nonequivalent) frame of reference. This is a transition from the frame of reference connected with the manifold with unclosed metric forms to the frame of reference connected with pseudostructure. [An example of a degenerate transformation is a transition from the Lagrange function to the Hamilton function. The transition from the Lagrange function $L$ to the Hamilton function $H$ (the transition from variables $q_{j}, \dot{q}_{j}$ to variables $q_{j}, p_{j}=\partial L / \partial \dot{q}_{j}$ ) is the transition from the tangent (Lagrangian) non-integrable manifold to integral structures forming the cotangent (Hamiltonian) manifold.]

\section{Nonidentical relation of the mathematical physics equations. Description of evolutionary processes in material media}

In the present Section the nonidentical relation will be obtained from the mathematical physics equations that describe material media such as thermodynamic, gas-dynamic, cosmic systems, the systems of charged particles and so on. It will be shown an unique role of nonidentical relations and degenerate transformations when describing evolutionary processes in material media.

\subsection{Nonidentical relation of the mathematical physics equations}

It is known that the equations of mathematical physics for material systems (material media) such as the thermodynamical, gasdynamical, cosmologic systems, the systems of charged particles and others consist of the equations of conservation laws for energy, linear momentum, angular momentum, and mass [6,7, 8] (In Appendix 2 some examples of such equations are presented.)

The nonidentical relation of the mathematical physics equations is obtained when studying the integrability of these equations. (This studying was carried out in a series of author's papers $[4,9,10]$. In present paper the accent will be made on a role of nonidentical relations and degenerate transformations in description of evolutionary processes.

The integrability of the mathematical physics equations depends on the consistency of conservation law equations. (It should be noted that the integrability of differential equations also depends on the consistency of derivatives along different directions. But here this problem will be not considered.)

Analysis of consistency of the conservation law equations. Nonidentical relation for the state functionals

Let us analyze the correlation of the equations that describe the conservation laws for energy and linear momentum.

We introduce two frames of reference: the first is an inertial one (this frame of reference is not connected with the material system), and the second is an accompanying one (this frame of reference is 
connected with the manifold made up by trajectories of the material system elements).

In the inertial frame of reference the energy equation can be reduced to the form:

$$
\frac{D \psi}{D t}=A_{1}
$$

here $D / D t$ is the total derivative with respect to time, $\psi$ is the functional of the state that specifies the material system, $A_{1}$ is the quantity that depends on specific features of material system and on external energy actions onto the system. [The action functional, entropy can be regarded as examples of the functional $\psi$. Thus, the equation for energy presented in terms of the action functional $S$ has a similar form: $D S / D t=L$, where $\psi=S, A_{1}=L$ is the Lagrange function. In mechanics of continuous media the equation for energy of ideal gas can be presented in the form [9]: $D s / D t=0$, where $s$ is the entropy.]

In the accompanying frame of reference the total derivative with respect to time is transformed into the derivative along the trajectory. Equation of energy is now written in the form

$$
\frac{\partial \psi}{\partial \xi^{1}}=A_{1}
$$

Here $\xi^{1}$ is the coordinate along the trajectory.

In a similar manner, in the accompanying reference system the equation for linear momentum appears to be reduced to the equation of the form

$$
\frac{\partial \psi}{\partial \xi^{v}}=A_{v}, \quad v=2, \ldots
$$

where $\xi^{v}$ are the coordinates in the direction normal to the trajectory, $A_{v}$ are the quantities that depend on specific features of material system and on external force actions.

Eqs. (11) and (12) can be convoluted into the relation

$$
d \psi=A_{\mu} d \xi^{\mu}, \quad(\mu=1, v)
$$

where $d \psi$ is the differential expression $d \psi=\left(\partial \psi / \partial \xi^{\mu}\right) d \xi^{\mu}$. Relation (13) can be written as

$$
d \psi=\omega
$$

here $\omega=A_{\mu} d \xi^{\mu}$ is the skew-symmetric differential form of the first degree. (A summing over repeated indices is carried out.) (In Appendix 3 examples of the quantities $A_{\mu}$ of nonidentical relation for the Euler and Navier-Stokes equations equations are presented.) Relation (14) has been obtained from the equation of the conservation laws for energy and linear momentum. In this relation the form $\omega$ is that of the first degree. If the equations of the conservation laws for angular momentum be added to the equations for energy and linear momentum, this form will be a form of the second degree. And in combination with the equation of the conservation law for mass this form will be a form of degree 3 . In general case the evolutionary relation can be written as

$$
d \psi=\omega^{p}
$$

where the form degree $p$ takes the values $p=0,1,2,3$. (The relation for $p=0$ is an analog to that in the differential forms, and it was obtained from the interaction of energy and time.) [A concrete form of relation (15) and its properties in the case of the Euler and Navier-Stokes equations were considered in paper [9] Relation (15) for $p=2$ were considered for electromagnetic field in paper [4] and in paper http://arxiv.org/pdf/math-ph/0310050v1.pdf]

Since the conservation law equations are evolutionary ones, the relation obtained is also evolutionary relation.

The evolutionary relation (15) was obtained with respect to the accompanying frame of reference, which is connected with the manifold made up by the trajectories of the material system elements This means that the skew-symmetric form in the evolutionary relation is defined on the manifold made up by trajectories of the material system elements, which is a deforming manifold. In Section 1 it was shown that the skew-symmetric form defined on such a manifold is evolutionary form which appears to be unclosed. Hence, the obtained evolutionary relation, which contains unclosed evolutionary form, proves to be nonidentical relation. In the next Subsection it will be shown a role of nonidentical evolutionary relation in description of evolutionary processes in material media and its mathematical and physical meaning will be disclosed.

[I should be emphasize that the nonidentical relation was obtained from the analysis of consistency of the conservation law equations. It may be noted that in papers by Pauli and Einstein $[8,12]$ the conservation law equations for energy and momentum, as well as the continuity equation, were studied, but only the properties of invariance of these equation were investigated. However, they didn't put a question on consistency of the conservation law equations. The presence of the energy-momentum tensor in Einstein's equation supposes that the conservation law equations for energy and momentum are consistent identically. However, the consistence of conservation law equations (due to non-commutativity of conservation laws) is fulfilled only discretely. In particular, this means that the energy-momentum tensor is fulfilled only discretely. And this imposes restrictions on Einstein's equation.]

\subsection{Mathematical and physical meaning of nonidenti- cal relation obtained from the equations for material medium}

The nonidentical evolutionary relation obtained from the mathematical physics equations, which describe material media, firstly, discloses the properties of the solutions to the mathematical physics equations, and, secondly, describes the material system state.

\section{Properties of the solutions to mathematical physics equa- tions}

The nonidentity of the evolutionary relation points to the fact that the conservation law equations turn out to be inconsistent. This means that the initial set of equations of mathematical physics proves to be nonintegrable (it cannot be convoluted into identical relation for differentials and be integrated). That is, the solutions to the mathematical physics equations are not functions (they will depend on the commutator of the form $\omega^{p}$ ). This also points to the fact that the tangent manifold, on which the solutions are defined, is not integrable.

As it follows from the properties of nonidentical relation, the closed dual form is obtained under degenerate transformation, and this points out to the realization of integral structure (pseudostructure) and closed inexact (only on pseudostructure) exterior form. In this case the identical relation is obtained from the nonidentical relation. This will point out to a consistency of the conservation law equations and an integrability of the mathematical physics equations. This points out to the fact that there are realized the solutions to the initial mathematical physics equations which are functions. These are so called generalized solutions, which are discrete functions. (The realization of integral structure (pseudostructure) with a closed exterior form points to emergence of a differential-geometrical structure. The physical structures, on which the conservation laws are fulfilled, are just such structures.)

Thus, from the nonidentical relation it follows that the mathematical physics equation have a double solution [10]. Below it will be shown a physical meaning of such double solutions.

\section{Physical meaning of nonidentical evolutionary relation. Description of the state of material medium}

Physical meaning of nonidentical evolutionary relation consists in the fact that it can describe the state of material medium since this relation contains the state functional, which specifies the material system state. [It should be noted that to every material medium its own state 
functional corresponds [11]. Thus, for thermodynamic system its state functional is the entropy, nonidentical which depends on thermodynamic variables, whereas for gasdynamic system its functional is the entropy, which depends on space-time variables [9]. It was shown above that for Lagrange system the action functional is a state functional For the system of charged particles and cosmological system a role of state functionals play, respectively, the Pointing vector [4] (in this case $p=2$ ) and Einstein's function.] But here there is some delicate matter.

Although the evolutionary relation includes the state functional (which specifies the material medium state), but, since this relation is nonidentical one, from this relation one cannot get the differential of the state functional $d \psi$. This points out to the absence of the state function and means that the material medium is in the non-equilibrium state.

Such non-equilibrium state is described by the solution to the mathematical physics equations, which is not a function.

The non-equilibrium means that some internal force acts in material medium. It is evident that the internal force is described by the commutator of skew-symmetric form $\omega^{p}$. (Everything that gives a contribution into the commutator of evolutionary form $\omega^{p}$ leads to emergence of internal forces that causes the non-equilibrium state of material medium (see [9]).)

Another property of the nonidentical evolutionary relation, namely, its self-variation, points out to the fact that the non-equilibrium state of material medium turns out to be self-varying. State of material medium changes but in this case it remains to be non-equilibrium during this process, since the evolutionary relation remains to be nonidentical during the process of self-variation.

The realization of identical relation from the evolutionary one points out to the transition of material medium to a localli-equilibrium state.

The solution to initial equations, which is a generalized one, i.e., a discrete function, just describes such localli-equilibrium state of material medium.

From identical relation one can define the differential of the state functional, and this points out to a presence of the state function and the transition of material medium from non-equilibrium state into equilibrium one. However, such a state of material medium turns out to be realized only locally due to the fact that differential of the state functional obtained is an interior differential (only on pseudostructure). And yet the total state of material medium remains to be non-equilibrium state because the evolutionary relation, which describes the material medium state, remains nonidentical one. (That is, there exists a duality. Nonidentical evolutionary relation acts simultaneously with identical relation.)

[It may be noted that these results show that the functionals of evolutionary relation are actually state functionals.]

The transition from non-equilibrium state to locally-equilibrium state means that nonmeasurable quantity, which is described by the commutator and act as internal force, converts into a measurable quantity of material medium.

This reveals in the emergence of some observed formations in material medium. Waves, vortices, fluctuations, turbulent pulsations, and so on are examples of such formations. The intensity of such formations is controlled by a quantity accumulated by the evolutionary form commutator. (This discloses a mechanism of such processes like an origin of vortices and turbulence $[9,10]$.)

Thus it is evident that the nonidentical evolutionary relation, firstly, discloses the properties of the solutions to the mathematical physics equations, namely, their double, and, secondly, describes the material medium state and the transition from non-equilibrium state to localliequilibrium one, and this process is accompanied by by emergence of physical structures and observable formations.

\section{Connection between the field-theory equa- tions and nonidentical relations obtained from the mathematical physics equations, which de- scribe material media.}

The nonidentical relations, from which closed exterior forms, i.e., differentials, are obtained, in essence, are the equations for obtaining differentials. (For differential equations the nonidentical relations, in essence, are equations for obtaining generalized solutions (eigenfunctions) to initial differential equations, and this corresponds to a realization of integrability of these equations.)

Below it will be shown that the field-theory equations are nonidentical relations obtained from the mathematical physics equations, which describe material media.

Correspondence between the nonidentical relations of the mathematical physics equations and the field-theory equations

Specific feature of the field-theory equations consists in the fact that the field-theory equations are equations for functionals such as a wave function, action functional, Einstein's tensor and so on. The Pointing vector is such a functional for the equations of electromagnetic field. (Entropy is such a functional for the fields generated by thermodynamical and gas-dynamical systems.)

As has been shown, the nonidentical evolutionary relation obtained from the mathematical physics equations for material media is a nonidentical relation for all these functionals.

Above it was shown that the nonidentical relations generate closed inexact exterior forms.

As it is known, the closed inexact exterior or dual forms (corresponding to the conservation laws) are also the solutions to the field-theory equations.

To the connection between the field-theory equations and nonidentical relations it also points out the fact that the field-theory equations are nonidentical relations. This is explained by the statement that only the equations that have the form of relations (nonidentical) may have the solutions which are differentials rather then functions. (As it is known, unlike the differential equations the solutions of which are functions, the field-theory equations have the solutions that are closed exterior forms, namely, differentials.)

One can verify that all equations of field theories, as well as the evolutionary relation, are nonidentical relations in differential forms or in the forms of their tensor or differential (i.e. expressed in terms of derivatives) analogs. For example,

- the Einstein equation is a relation in differential forms;

- the Dirac equation relates Dirac's bra- and cket-vectors, which made up a differential form of zero degree;

- the Maxwell equations have the form of tensor relations;

- the Schrődinger's equations have the form of relations expressed in terms of derivatives and their analogs.

From the field-theory equations, as well as from the nonidentical evolutionary relation, the identical relation, which contains the closed exterior form, is obtained. As one can see, from the field-theory equations it follows such identical relations as the Poincare invariant, which connects closed exterior forms of first degree; the relations $d \theta^{2}=0, d^{*} \theta^{2}=0$ are those for closed exterior forms of second degree obtained from Maxwell equations; the Bianchi identity for gravitational field.

Thus, one can see that there exists a correspondence between the field-theory equations, which describe physical fields, and the nonidentical evolutionary relation obtained from the equations of mathematical physics for a material medium.

Connection between the field-theory equations and the equations of mathematical physics which describe material media 
A correspondence between the nonidentical relation and the fieldtheory equations points out to a connection between the field-theory equations and the equations of mathematical physics which describe material media.

Such a connection, which is common to all field theories, discloses the general foundations of field theories. This connection has to be taken into account when building a general field theory.

It should be emphasized that a possibility to clarify the problems of field theories is based on the properties of conservation laws.

As it is known (and was noted above) the equations of mathematical physics, which describe material media, consist of the equations of conservation laws for energy, linear momentum, angular momentum, and mass, which are conservation laws for material media. The conservation laws for material systems establish a balance between the change of physical quantities and the external action. Such conservation laws are described by differential equations.

In field theory "the conservation laws" are those that claim an existence of conservative quantities or objects. The conservation laws for physical fields are such conservation laws. They are described by closed exterior skew-symmetric forms. (The Noether theorem is an example.)

Generating closed exterior forms, which correspond to conservation laws for physical fields, by nonidentical relations obtained from the conservation law equations for material media, discloses the connection between the conservation laws for physical fields and for material systems and their controlling role in evolutionary processes.

\section{Conclusion}

In the paper it was shown an unique role of nonidentical relations of skew-symmetric differential forms in mathematics and mathematical physics. This relates to the fact that nonidentical relations generate closed exterior forms which are differentials. Nonidentical relations discloses hidden properties of the equations of mathematical physics that enables one to describe discrete transitions, processes of emergence of physical structures and observable formations (waves, vortices, turbulent pulsations, and so on) and gives a possibility to understand the foundations of the field-theory equations, namely, their connection to the mathematical physics equations for material media.

\section{Some properties of manifolds}

Appendix 1

Assume that on a manifold one can place a coordinate system with base vectors $\mathbf{e}_{\mu}$ and define the metric forms for a manifold [13]: $\left(\mathbf{e}_{\mu} \mathbf{e}_{v}\right),\left(\mathbf{e}_{\mu} d x^{\mu}\right),\left(d \mathbf{e}_{\mu}\right)$.

If a metric form is closed (i.e., its commutators equal zero), then this metric is defined by $g_{\mu v}=\left(\mathbf{e}_{\mu} \mathbf{e}_{v}\right)$ and the results of a translation over a manifold of the point $d \mathbf{M}=\left(\mathbf{e}_{\mu} d x^{\mu}\right)$ and of the unit frame $d \mathbf{A}=\left(d \mathbf{e}_{\mu}\right)$ prove to be independent of the the path of integration. Such a manifold is integrable. [On the integrability of manifolds, see[13]]. If metric forms are nonclosed (the commutators of metric forms are nonzero), this points to the fact that this manifold is nonintegrable. Metric forms and their commutators define the metric and differential characteristics of a manifold. Closed metric forms define a manifold structure, i.e. the internal characteristics of a manifold. And, nonclosed metric forms define the differential characteristics of a manifold. The commutators of nonclosed metric forms define the manifold differential characteristics that specify the manifold deformations: bending, torsion, rotation, twist. Thus, the final result is, that nonintegrable manifolds, i.e. the manifolds with nonclosed metric forms, are deformed manifolds.

To describe manifold differential characteristics and, correspondingly, metric form commutators, one can use connectedness. If the components of a metric form can be expressed in terms of connectedness $\Gamma_{\mu v}^{\rho}$ [13], the expressions $\Gamma_{\mu v}^{\rho},\left(\Gamma_{\mu v}^{\rho}-\Gamma_{v \mu}^{\rho}\right)$ and $R_{v \rho \sigma}^{\mu}$ are components of the commutators of metric forms of zeroth- first- and third degrees. As is known, a commutator of the zeroth degree metric form $\Gamma_{\mu \nu}^{\rho}$ characterizes the bend, while that of the first degree form $\left(\Gamma_{\mu v}^{\rho}-\Gamma_{v \mu}^{\rho}\right)$ characterizes the torsion, the commutator of the third degree metric form $R_{v \rho \sigma}^{\mu}$ determines the curvature. In the case of nonintegrable manifolds, the components of the metric form commutators are nonzero. In particular, the connectednesses $\Gamma_{\mu v}^{\rho}$ are not symmetric. (For manifolds with a closed metric form of the first degree, the connectednesses are symmetric.) The difference between exterior and evolutionary forms depends on the properties of metric forms of the manifold on which these skew-symmetric forms are defined. The peculiarity of skew-symmetric forms defined on nonintegrable manifold can be demonstrated by the example of a skew-symmetric form of first-degree. Let us consider the first-degree form $\omega=a_{\alpha} d x^{\alpha}$. The differential of this form can be written as $d \omega=$ $K_{\alpha \beta} d x^{\alpha} d x^{\beta}$, where $K_{\alpha \beta}=a_{\beta ; \alpha}-a_{\alpha ; \beta}$ are components of the commutator of the form $\omega$, and $a_{\beta ; \alpha}, a_{\alpha ; \beta}$ are covariant derivatives. If we express the covariant derivatives in terms of connectedness (if it is possible), they can be written as $a_{\beta ; \alpha}=\partial a_{\beta} / \partial x^{\alpha}+\Gamma_{\beta \alpha}^{\sigma} a_{\sigma}$, where the first term results from differentiating the form coefficients, and the second term results from differentiating the basis. If we substitute the expressions for covariant derivatives into the formula for commutator components, we obtain the following expression for commutator components of the form $\omega$ :

$$
K_{\alpha \beta}=\left(\frac{\partial a_{\beta}}{\partial x^{\alpha}}-\frac{\partial a_{\alpha}}{\partial x^{\beta}}\right)+\left(\Gamma_{\beta \alpha}^{\sigma}-\Gamma_{\alpha \beta}^{\sigma}\right) a_{\sigma}
$$

Here the expressions $\left(\Gamma_{\beta \alpha}^{\sigma}-\Gamma_{\alpha \beta}^{\sigma}\right)$ entered into the second term are just components of the commutator of the first-degree metric form that specifies the manifold deformation and hence is nonzero. (In the commutator of exterior form, which is defined on integrable manifold, the second term absents: the connectednesses are symmetric, that is, the expression $\left(\Gamma_{\beta \alpha}^{\sigma}-\Gamma_{\alpha \beta}^{\sigma}\right)$ vanishes).

The evolutionary differential form commutator, in contrast to that of the exterior one, cannot be equal to zero because it involves the metric form commutator being nonzero Since the commutator, and hence the differential, of skew-symmetric form defined on nonintegrable manifold are nonzero, this means that such a form, in contrast to the case of the exterior form, cannot be closed one.

The equations of mathematical physics describing material media.

Appendix 2 Such equations are presented for example in paper [8] (paragraphs 30,37). The equations of gas-dynamics, which describe the flow of ideal gas, are the example of such equations. They can be written in the form [6]:

The conservation law equations for energy (see 5.3.21)

$$
\rho \frac{D}{D t}\left(e+\frac{1}{2} u^{2}\right)=f_{1}
$$

The conservation law equations linear momentum (see 5.3.15):

$$
\rho \frac{\partial u_{j}}{\partial t}+\rho u_{k} \frac{\partial u_{j}}{\partial x_{k}}=f_{j}
$$

The conservation law equations for mass (see 5.3.12)

$$
\frac{\partial \rho}{\partial t}+\frac{\partial}{\partial x_{k}}\left(\rho u_{k}\right)=0
$$

Appendix 3

Examples of the quantities $A_{\mu}$ of nonidentical relation for the Euler and NavierStokes equations

In the case of viscous heat-conducting gas described the Navier-Stokes equations the expression $A_{1}$ can be written as (see [6], Chapter 6, formula (6.2.4))

$$
A_{1}=\frac{1}{\rho} \frac{\partial}{\partial x_{i}}\left(-\frac{q_{i}}{T}\right)-\frac{q_{i}}{\rho T} \frac{\partial T}{\partial x_{i}}+\frac{\tau_{k i}}{\rho} \frac{\partial u_{i}}{\partial x_{k}}
$$

Here $q_{i}$ is the heat flux and $\tau_{k i}$ is the viscous stress tensor.

In the case of ideal gas described by the Euler equations we have $A_{1}=0$.

In the case of two-dimensional flow of ideal gas one can obtain the following expression for the coefficient $A_{v}$ (see [6], Chapter 6, formula (6.7.12)):

$$
A_{v}=\frac{\partial h_{0}}{\partial v}+\left(u_{1}^{2}+u_{2}^{2}\right)^{1 / 2} \zeta-F_{v}+\frac{\partial U_{v}}{\partial t}
$$

where $\zeta=\partial u_{2} / \partial x-\partial u_{1} / \partial y$

In the case of viscous gas the expression $A_{v}$ includes additional terms related to viscosity and heat-conductivity.]

\section{References}

[1] Cartan E., Les Systemes Differentials Exterieus ef Leurs Application Geometriques, Paris, Hermann, (1945).

[2] Petrova L.I., Exterior and evolutionary differential forms in mathematical physics: Theory and Applications, Lulu.com, (2008).

[3] Petrova L.I., "Role of skew-symmetric differential forms in mathematics", http://arxiv.org/pdf/1007.4757vl.pdf, (2010).

[4] Petrova L.I., A new mathematical formalism: Skew-symmetric differential forms in mathematics, mathematical physics and field theory, URSS, Moscow, (2013).

[5] Bott R., Tu L.W., Differential Forms in Algebraic Topology, Springer, $N Y,(1982)$.

[6] Clarke J.F., Machesney M., The Dynamics of Real Gases, Butterworths, London, (1964).

[7] Tolman R.C., Relativity, Thermodynamics, and Cosmology, Clarendon Press, Oxford, UK, (1969).

[8] Pauli W., Theory of Relativity, Pergamon Press, (1958).

[9] Petrova L., "The Peculiarity of Numerical Solving the Euler and NavierStokes Equations", American Journal of Computational Mathematics, Vol.4, No. 4, (2013), pp.305-310.

[10] Petrova L.I., "Hidden properties of the equations of mathematical physics. Evolutionary relation for the state functionals and its connection with the field-theory equations", Journal of Progressive Research in Mathematics(JPRM), Vol.4, No. 2, (2015), pp. 309-320.

[11] Petrova L.I., "Physical meaning and a duality of concepts of wave function, action functional, entropy, the Pointing vector, the Einstein tensor", Journal of Mathematics Research, Vol. 4, No. 3, (2012), pp.78 88.

12] Einstein A., The Meaning of Relativity, Princeton, (1953).

[13] Tonnelat M.-A., Les principles de la theorie electromagnetique et la relativite, Masson, Paris, (1959). 